\title{
Criação de sistema interno on-line para notificação de incidentes no espaço
}

\section{hospitalar: Relato de experiência}

\author{
Creation of an internal online system for notification of incidents in the hospital space: Experience \\ report
}

Creación de un sistema interno online de notificación de incidencias en el espacio hospitalario: Informe de experiencia

Recebido: 16/04/2021 | Revisado: 25/04/2021 | Aceito: 06/05/2021 | Publicado: 19/05/2021

\author{
Alice Eulália Chagas Ribeiro \\ ORCID: https://orcid.org/0000-0001-6068-9354 \\ Universidade Federal do Estado do Rio de Janeiro, Brasil \\ E-mail: aliceeulalia.ppgsteh@gmail.com \\ Cristiane de Oliveira Novaes \\ ORCID: https://orcid.org/0000-0002-5272-3759 \\ Universidade Federal do Estado do Rio de Janeiro, Brasil \\ E-mail: novaes.cristiane@gmail.com
}

\begin{abstract}
Resumo
O objetivo deste artigo foi relatar a criação de um sistema interno de notificação de incidentes on-line no espaço hospitalar, desenvolvido no âmbito do Núcleo de Segurança do Paciente da instituição. Metodologia: A construção do sistema on-line foi dividida em etapas, sendo a primeira a definição da construção do sistema on-line interno com base na interface FormSUS. A etapa seguinte é realizada por um gerente de formulários FormSUS profissional, outro respondente profissional nomeado e um monitor. O acesso responsável respeita os preceitos éticos e os dados individuais identificados, garantindo privacidade e confidencialidade. O formulário é restrito e só pode ser acessado a convite do gestor. Resultados e discussão: O formulário FormSUS pode ser acessado por meio de um atalho que é exibido em todos os computadores. O gestor profissional é o técnico responsável pela criação e/ou desenho da estruturação de formulários específicos para atender às necessidades do processo de notificação de incidentes. O respondente notifica as ocorrências por meio de um atalho que leva ao próprio formulário, já estruturado, para enviar aos profissionais do Núcleo de Segurança do Paciente. O conteúdo de cada formulário e o rastreamento de suas respostas são de responsabilidade do gestor técnico de criação do formulário e de sua equipe. O formulário permite a inclusão de dados do paciente e do incidente: nome completo do paciente; número do histórico médico; enfermaria e leito; data de internação; data de incidência; grupo de idade; sexo; descrição do incidente; identificação do notificador; contato, email e telefone; escritório ou função. Considerações finais: A construção de um sistema interno de notificação de incidentes on-line pode representar maior agilidade na detecção e tratamento dos incidentes, maior aderência às notificações por parte dos profissionais, além de melhorar a segurança e a qualidade das ações no atendimento ao paciente.
\end{abstract}

Palavras-chave: Segurança do paciente; Eventos adversos; Erros médicos; Notificação; Tecnologia em saúde.

\begin{abstract}
The article was to report the creation of an internal online incident notification system in the hospital space, developed in the context of the institution's Patient Safety Center. Methodology: The construction of the online system was divided into stages, the first being the definition of the construction of the internal online system based on the FormSUS interface. The next step is performed by a professional FormSUS forms manager, another named professional respondent, and a monitor. Responsible access respects the ethical precepts and the individual data identified, guaranteeing privacy and confidentiality. The form is restricted and can only be accessed at the invitation of the manager. Results and Discussion: The FormSUS form can be accessed through a shortcut that is displayed on all computers. The professional manager is the technician responsible for the creation and / or design of the structuring of forms, specific to meet the needs of the incident notification process. The respondent notifies the incidents through a shortcut that leads to the form itself, already structured, to send to the professionals of the Patient Safety Center. The content of each form and the tracking of your responses are the responsibility of the technical director of creating the form and his team. The form allows the inclusion of patient and incident data: full name of the patient; Medical history number; infirmary and bed; date of hospitalization; incidence date; age group; sex; description of the incident; identification of the notifier; contact, email and phone; office or function. Final considerations: The construction of an
\end{abstract}


internal online incident notification system may represent greater agility in the detection and handling of incidents, greater adherence to notifications by professionals, in addition to improving security and quality actions in customer service patient.

Keywords: Patient safety; Adverse events; Medical errors; Notification; Health technology.

\section{Resumen}

El propósito de este artículo fue reportar la creación de un sistema interno en línea de notificación de incidencias en el espacio hospitalario, desarrollado en el ámbito del Centro de Seguridad del Paciente de la institución. Metodología: La construcción del sistema online se dividió en etapas, siendo la primera la definición de la construcción del sistema online interno basado en la interfaz FormSUS. El siguiente paso lo lleva a cabo un gestor profesional de formularios FormSUS, otro profesional nombrado encuestado y un monitor. El acceso responsable respeta los preceptos éticos y los datos individuales identificados, garantizando la privacidad y confidencialidad. El formulario está restringido y solo se puede acceder a él por invitación del gerente. Resultados y discusión: Se puede acceder al formulario FormSUS a través de un o acceso directo que se muestra en todas las computadoras. El gerente profesional es el técnico responsable de la creación y / o diseño de la estructuración de los formularios, específicos para atender las necesidades del proceso de notificación de incidencias. El encuestado notifica las incidencias mediante un atajo o que conduce al propio formulario ya estructurado para enviar a los profesionales del Centro de Seguridad del Paciente. El contenido de cada formulario y el seguimiento de sus respuestas son responsabilidad del director técnico de creación del formulario y su equipo. El formulario permite incluir datos del paciente y del incidente: nombre completo del paciente; numero de historia clínica; enfermería y cama; fecha de hospitalización; fecha de ocurrencia; grupo de edad; sexo; descripción del incidente; identificación del notificador; contacto, correo electrónico y teléfono; oficina o función. Consideraciones finales: La construcción de un sistema interno online de notificación de incidencias puede representar mayor agilidad en la detección y manejo de incidencias, mayor adherencia a las notificaciones por parte de los profesionales, además de mejorar las acciones de seguridad y calidad en la atención al paciente.

Palabras clave: Seguridad del paciente; Eventos adversos; Errores médicos; Notificación; Tecnologia de la salud.

\section{Introdução}

O Ministério da Saúde instituiu o Programa Nacional de Segurança do Paciente através da Portaria $n^{\circ} 529$, de $1^{\circ}$ de abril de 2013, a fim de melhorar a qualidade dos processos do cuidado, promover a segurança do paciente, a gestão de risco, a implantação dos Núcleos de Segurança do Paciente, incentivar a participação de pacientes e familiares no cuidado e facilitar o acesso a informações referentes à segurança do paciente e à promoção da cultura de segurança. Para tal, recomendou a inclusão do tema no ensino, na graduação, pós-graduação e pesquisa (Ministério da Saúde, 2013). O documento de referência para o Programa Nacional de Segurança do Paciente versa sobre a implantação de ações, prevenção de riscos e redução de incidentes, desde a admissão, transferência, alta e óbito.

Nesse campo, diversos países, no intuito de melhorar a segurança dos pacientes, têm adotado estratégias para aumentar as notificações de incidentes (Sousa et al., 2019). O principal objetivo dos sistemas de notificação dos incidentes é ampliar a produção e a disseminação de informações referentes às ocorrências, para que os dados sejam submetidos a uma análise, e, com as informações obtidas, sejam implementados procedimentos que promovam o refreamento de tais eventualidades. Considerando a aprendizagem e melhoria dos processos, os sistemas de notificação de incidentes devem prover recursos adequados, apoio organizacional com definições claras e ações colaborativas. As notificações exigem o envolvimento dos profissionais como condição sine qua non para a melhoria das estruturas e processos que possam atenuar a ocorrência de incidentes (Wachter \& Gupta, 2018).

Neste estudo, foram adotados os termos incidente com dano e evento adverso como sinônimos, conforme as definições para os principais termos relacionados na Classificação Internacional de Segurança do Paciente (World Health Organization, 2009), sendo o incidente todo evento ou circunstância que poderia ter causado ou resultou em dano desnecessário ao paciente. O sistema de notificação de incidentes permite que os profissionais de saúde registrem, de forma voluntária ou confidencial, a ocorrência de incidentes, com o objetivo de aumentar o conhecimento sobre erros e riscos. Por meio da investigação e análise dos incidentes, geram-se informações e correções das fragilidades e falhas (Meyer-Masseti et al., 2011). 
No Brasil, a notificação de incidentes é obrigatória, de acordo com a Resolução da Diretoria Colegiada n 36/2013 (Agência Nacional de Vigilância Sanitária, 2013). Trata-se de uma ferramenta reativa para a gestão de risco, vigilância e monitoramento do que é notificado - essenciais para que ações específicas sejam praticadas pelos profissionais de saúde, cruciais para a aprendizagem e para a qualificação da segurança do paciente (Sousa et al., 2019).

Segundo Santa Rosa e Moraes, o objetivo principal da área de interação humano-computador é o projeto e o desenvolvimento de sistemas com o propósito de melhorar a eficácia e proporcionar satisfação. Testar a usabilidade de sistemas significa ter certeza de que as pessoas podem reconhecer e interagir com funções que satisfaçam suas necessidades (Dumas \& Redish, 1999 apud Santa Rosa \& Moraes, 2008, p. 14).

Nesse sentido, busca-se com o presente estudo realizar um relato de experiência sobre a criação de um sistema interno on-line para notificação de incidentes no âmbito de um hospital público federal (Lima et al., 2020); desenvolvido como um desdobramento de um projeto de mestrado do Programa de Pós-Graduação em Saúde e Tecnologia no Espaço Hospitalar da Universidade Federal do Estado do Rio de Janeiro. Na referida instituição, as notificações de ocorrências eram realizadas de maneiras distintas, como comunicação oral e depósito em urnas. A proposta de implementação de um sistema on-line no âmbito do hospital federal parte da demanda por um serviço mais prático e eficiente, dada a facilidade e agilidade que a internet oferece, organizando atividades e compartilhando arquivos de forma confidencial, colaborando com a disseminação de informações de interesse da saúde. Desta forma, evidenciam-se os desdobramentos da proposta e recorre-se a Santa Rosa e Moraes, para abarcar a discussão acerca da Interação Humano-Computador, também denominada IHC ou HCI (Human-Computer Interaction).

\section{Metodologia}

Trata-se de um relato de experiência sobre a criação de um sistema interno on-line para notificação de incidentes em hospital público federal (Lima et al., 2020), desenvolvido no escopo do Núcleo de Segurança do Paciente da instituição, como um desdobramento de um projeto de mestrado do Programa de Pós-Graduação em Saúde e Tecnologia no Espaço Hospitalar da Universidade Federal do Estado do Rio de Janeiro.

Para realizar a construção do sistema, foram operacionalizadas as seguintes etapas: definir o FormSUS como a interface a ser utilizada, um profissional gestor cadastrado para operacionalizar o formulário, um profissional respondente e um monitor. O manual de instruções é específico para a construção de formulários aplicados em diversas finalidades, contendo informações pertinentes à adaptação do sistema para estruturar o sistema de notificações.

Como material fundamental da metodologia, recorre-se ao Manual do Gestor do FormSUS e suas diretrizes organizacionais. A construção do sistema on-line necessita somente de computador, rede de internet, um navegador, o logo para sinalizar o atalho, o manual em si e uma conta de e-mail para servir o gestor e o respondente. Considerando, portanto, que não há custos adicionais para a estruturação do sistema interno on-line.

A implementação do sistema on-line parte da necessidade de um serviço mais colaborativo, no que se refere ao compartilhamento de informações dos incidentes. Desta forma, a internet e suas atribuições apresentam-se como importante ferramenta, pois permitem o acesso a informações e a transferência de dados por meio da Trasmission Control Protocol ou Protocolo de Controle de Transmissão (TCP) e Internet Protocol, também conhecido como Protocolo de Internet (IP) (infra). Por oferecer esses recursos, a internet passou a ser o meio de comunicação mais utilizado atualmente, sendo passível de aplicação em meios hospitalares como um facilitador do compartilhamento das notificações de incidentes, como a proposta deste estudo.

O uso da internet no âmbito hospitalar, cenário desta pesquisa, parte da prerrogativa de Interação Humano-Computador, também denominada IHC ou HCI (Human-Computer Interaction). Segundo Santa Rosa e Moraes, o objetivo principal da área de interação humano-computador é o desenvolvimento de sistemas com o intuito de aprimorar a eficácia e proporcionar 
satisfação ao seu usuário. O IHC tem como finalidade a melhoria de sistemas que podem oferecer usabilidade ao usuário, ou seja, proporcionar segurança e eficiência durante a execução das tarefas. Para que um produto ou serviço possa ser considerado com boa usabilidade, é necessário que ele contenha alguns aspectos, como facilidade de utilização eficiente.

[...] a usabilidade é um atributo de todo produto - como a funcionalidade. Testar a funcionalidade significa certificarse que o produto funciona de acordo com as especificações. [...] Testar a usabilidade significa ter certeza de que as pessoas podem reconhecer e interagir com funções que satisfaçam suas necessidades (Dumas \& Redish, 1999 apud Santa Rosa \& Moraes, 2008, p. 14).

Nesse sentido, a partir de pesquisa bibliográfica e de observações referentes ao funcionamento do sistema de comunicação digital, foram delimitados os problemas para análise de acordo com o sistema alvo apresentado, segundo modelagem sistêmica de Moraes e Mont'Alvão (2003). De acordo com as peculiaridades e necessidades do âmbito hospitalar, a partir de uma demanda de maior comunicação e compartilhamento de informações, optou-se pelo FormSUS, uma ferramenta do Departamento de Informática do Sistema Único de Saúde (DATASUS), que pode contribuir de modo fundamental para as diretrizes da Política Nacional de Informação e Informática, que tem, entre suas metas, a democratização das informações e a transparência na gestão pública. O serviço ofertado pelo FormSUS permite o "estabelecimento de mecanismos de compartilhamento de dados; a ampliação da produção e da disseminação de informações de saúde; o acesso livre a bases de dados em saúde não-identificados"; com usabilidade e "acesso responsável, respeitados os preceitos éticos, a dados individuais identificados, garantindo a privacidade e confidencialidade dos respondentes" (Departamento de Informática do Sistema Único de Saúde [DATASUS], 2021).

Atualmente, o FormSUS tem sido a interface amplamente empregada pelo Sistema Único de Saúde (SUS), e por órgãos públicos parceiros, para atividades de interesse público, e possibilita a criação de formulários na web. Suas normas de utilização são compatíveis com a legislação e com a Política de Informação e Informática do SUS, havendo a necessidade de cadastrar-se como Gestor de Formulário do FormSUS para criar formulários (DATASUS, 2021).

Este sistema foi desenvolvido para dar agilidade, estruturar e promover qualidade ao processo de coletar, disseminar dados pela internet mediante o fornecimento de formulários prontos, visando facilitar a sua construção. Pode exportar dados em arquivos nos formatos CSV - texto separado por vírgula - ou outro separador se necessário; XLS - formato Excel; DBF - Banco de Dados Dbase, e qualquer dúvida pode ser solucionada através da homepage: http://formsus.datasus.gov.br/. O instrumento foi inspirado em iniciativa anterior ao DATASUS, o programa "Gera HTM, Soluções na Web", disponível no Yahoo Groups e no serviço norte-americano

(Ministério da Saúde, 2015a).

Tarefas tornam-se mais complexas quando há necessidade de compartilhar dados ou acompanhar em tempo real os resultados; nesse sentido, o FormSUS viabiliza o desenvolvimento de formulários sem que se dependa de analistas e programadores. Ademais, permite criar um sistema, um banco de dados e gerar relatórios, sem consumir muito tempo, dando aos técnicos e profissionais a autonomia para criar e modificar formulários, por meio de uma interface simples.

O sistema armazena dados cadastrais, formulários e dados de formulários que estarão conectados no ambiente do DATASUS, seguindo as diretrizes do governo federal. O FormSUS foi desenvolvido em software livre, e usa banco de dados MYSQL 4 e linguagem PHP 5 (Ministério da Saúde, 2015a).

O formulário desenvolvido nesta pesquisa foi estruturado com base no FormSUS versão 3.0, que consiste na utilização de um instrumento já existente no Ministério da Saúde, adaptado para o processo de notificações on-line de incidentes no ambiente de trabalho. No sistema podem ser inseridas todas as informações necessárias referentes ao procedimento: identificação do paciente pelo nome completo, faixa de idade, sexo, número de prontuário, localização do leito e enfermaria, data da internação, 
data da ocorrência do incidente, descrição completa do incidente no espaço destinado para este fim, identificação do profissional notificador, contato por e-mail, telefone ou presencial, cargo e função do profissional notificador.

Essas informações são organizadas e inseridas no instrumento próprio para a notificação de um ou mais incidentes listados e disponíveis para a seleção do que será notificado. A listagem foi estabelecida segundo as seis metas internacionais de segurança do paciente, com a adição de uma meta 7 (úlceras por pressão), um item "não se aplica", eventos adversos, incidentes sem dano, circunstâncias notificáveis e quase falha. Os incidentes ou eventos serão marcados por um " $x$ " ou "o" para o notificar.

Os gestores definem quais os profissionais que receberão as informações, como parte operacional do processo, e quais profissionais podem conhecer o conteúdo delas. Estes estarão envolvidos na análise, caracterização, classificação, ações de gerenciamento, aprendizagem e na definição das propostas e soluções para a prevenção de incidentes junto aos profissionais envolvidos (Ministério da Saúde, 2015a).

A construção do sistema on-line teve como primeiro passo a definição um profissional Gestor de Formulário do FormSUS cadastrado, mas não como respondente do formulário. Cada gestor tem a responsabilidade de cuidar e garantir a aplicação de normas de produção, disseminação de informações de saúde e acesso livre por meio de bases de dados não identificados. O acesso responsável respeita os preceitos éticos acerca de dados individuais identificados, garantindo a privacidade e confidencialidade. O formulário é restrito e pode ser acessado somente a convite do gestor. Quanto ao armazenamento e à segurança de dados, as regras como backup e disponibilidade devem estar em acordo com as diretrizes e normas de segurança (Ministério da Saúde, 2015b).

O formulário do FormSUS pode ser acessado pelo link informado: http://formsus.datasus.gov.br/site/formulario.php?id _aplicacao= XXX, no qual XXX é o número do formulário e o link não deve ter acentos nem cedilha (ç).

Dessa forma, é de suma importância apresentar os responsáveis pelo processo e suas atribuições: 1. O Gestor do formulário: é o profissional técnico responsável pela criação do projeto do formulário. O gestor de formulário pode ser um técnico que ajudará a criar o formulário no sistema, ficando os membros da equipe responsáveis pela definição da finalidade e do conteúdo do formulário, e pela utilização dos dados que serão coletados; 2. Respondente: um respondente pode ser o gestor do formulário, desde que cadastrado no sistema. Este profissional terá acesso a todos os campos ou fichas preenchidas, mas não poderá alterar nem excluir nenhuma; 3. Monitor: possui as mesmas permissões do respondente e pode alterar os campos ou as fichas.

O conteúdo de cada formulário e o monitoramento de suas respostas são de responsabilidade do responsável técnico pela criação do formulário, ou de sua equipe gestora. Nenhum formulário será alterado sem autorização do responsável pelo formulário, o que está em conformidade com a Política e Normas de Uso do FormSUS e com as recomendações do Ministério da Saúde (DATASUS, 2021).

\section{Resultados e Discussão}

Antes de o Ministério da Saúde instituir o Programa Nacional de Segurança do Paciente e a Resolução da Diretoria Colegiada n 36/2013 (Agência Nacional de Vigilância Sanitária, 2013), as notificações eram realizadas na instituição, cenário deste estudo, de formas distintas: por meio de comunicação verbal, descrição de incidentes, em ordens de serviço, ofícios, depósito em urnas, por e-mail pessoal, segundo o fluxograma e o termo construídos como demonstrado na Figura 1 e no Quadro 1. 
Figura 1 - Fluxograma para notificar incidentes.
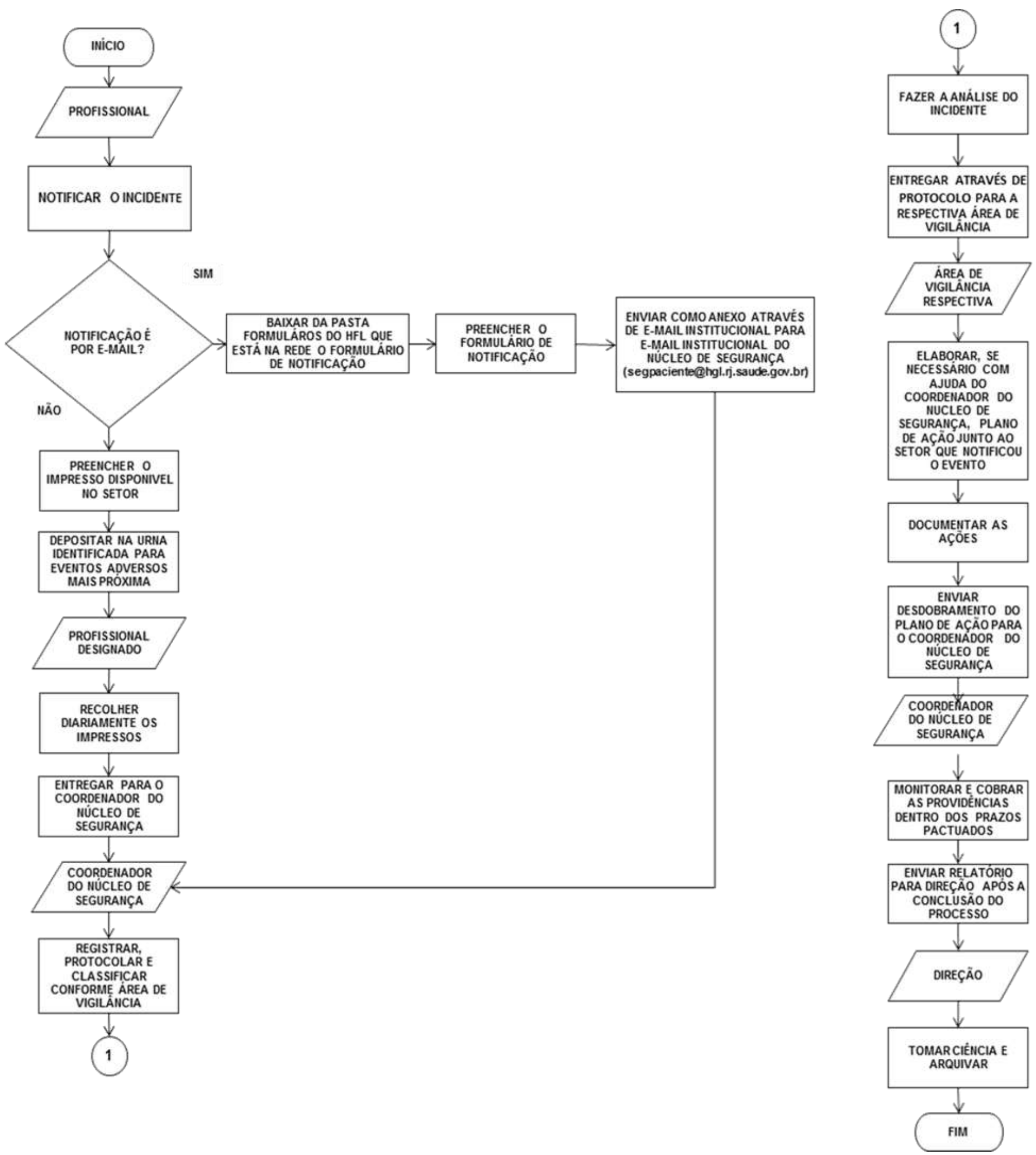

Fonte: Elaborado pelo Setor de Qualidade. 
Quadro 1 - Termo anterior para notificações.

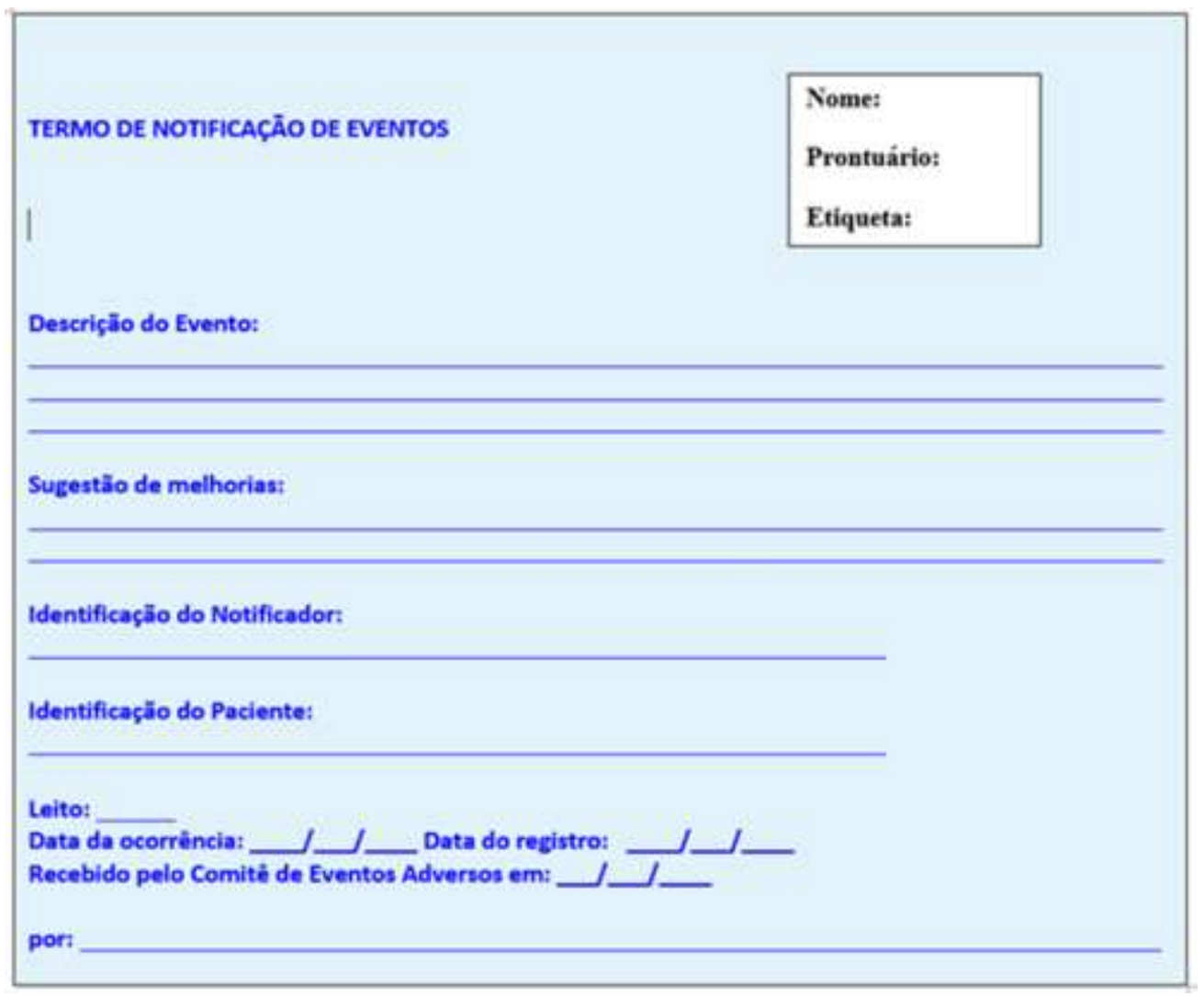

Fonte: Elaborado pelo Setor de Qualidade.

Observa-se que o processo de envio das notificações e descrições das ocorrências eram realizadas por meios diversos: depósito em urnas, comunicação verbal ou envio por e-mail pessoal, como supracitado e apresentado na Figura 1 e no Quadro 1, o que demonstra a complexidade dos registros dos incidentes. No entanto, nos últimos anos, nota-se a demanda crescente e concreta por um serviço mais prático e eficiente. Nesse sentido, as chamadas tecnologias emergentes trazem crescimento para este campo de atuação, e benefícios como o Prontuário Eletrônico do Paciente, o Business Intelligence, a certificação digital, as tecnologias sem fio e a computação móvel. Ou seja, em paralelo às tecnologias próprias da área, cada vez mais os profissionais de saúde adotam técnicas e conhecimentos de outras áreas, e atualmente, o processo de cuidado tem sido acompanhado pelas ferramentas derivadas das Tecnologias da Informação e Comunicação (TIC) (Instituto Brasileiro de Geografia e Estatística, 2009). Na Europa, América do Norte, Austrália e em outros países, a presença de tecnologias produz conhecimentos e dissemina a adoção tecnológica na área da saúde. E a participação nos processos de incorporação e a utilização de tecnologias contribuem para a equidade, o acesso, a maior eficiência e efetividade, e qualidade dos serviços e sustentabilidade (Banta \& Almeida, 2009).

A Organização Mundial da Saúde afirma que a saúde digital é o campo do conhecimento e da prática associados com qualquer aspecto de adoção de tecnologia digital para melhorar a saúde, desde o início até a operação dinâmica, e que progride rapidamente. A Estratégia de Saúde Digital no Brasil foi elaborada com base no pacote de ferramentas e nas experiências bemsucedidas em países como Canadá, Austrália, Inglaterra e Escócia (Organização Mundial da Saúde; União Internacional das Telecomunicações, 2012), tendo sido apresentada pelo Ministério da Saúde e denominada e-Saúde (Ministério da Saúde, 2017). Os principais objetivos do e-Saúde para o Brasil são identificar políticas, modelos de governança, financiamento, infraestrutura, tecnologias, recursos humanos e viabilizar o cenário estratégico. Por meio do e-Saúde, são obtidos benefícios que abarcam o Prontuário Eletrônico do Paciente, Telessaúde, Estratégia Saúde da Família, Registro Eletrônico de Saúde, Interoperabilidade, Picture Archiving and Communication System e Saúde na Nuvem (Hsia et al., 2019; Ministério da Saúde, 2017). 
Os recursos de TIC na saúde têm sido essenciais para facilitar as atividades e o uso de ferramentas para resolver problemas na área de saúde. Deve-se também considerar o fator humano para o sucesso de um sistema, sendo o treinamento um item indispensável no uso da tecnologia adotada (Novoa \& Valerio Netto, 2019). A adoção de indicadores permite monitorar e avaliar o uso das TIC, como gestão, infraestrutura, legislação, investimento etc. O TIC Saúde (https://cetic.br/pesquisas/) investiga a infraestrutura, a disponibilidade das TIC e de outras aplicações baseadas nos estabelecimentos de saúde no Brasil e o Global Digital Health Index faz uma análise comparativa das ações de saúde digital entre países (https://www.digitalhealthindex.org/).

Desse modo, o desenvolvimento de um sistema informatizado de notificações nas instituições converge com a tendência de estruturação dos cuidados em saúde, e pode facilitar e agilizar o processo de envio de informação para gestão de risco, bem como a tomada de decisões e, consequentemente, ampliar a qualidade da assistência e a segurança do paciente. Além disso, o sistema informatizado é uma importante estratégia de promoção da qualidade aliada à sustentabilidade. É partir disso que se propôs o sistema on-line, identificado por este símbolo: Rerrined bys. Seu acesso é feito por meio de um atalho nas telas dos computadores no ambiente de trabalho, que conduzirá diretamente ao formulário de notificações. O formulário estará disponível para notificar de forma digital e descritiva, havendo um espaço destinado à descrição e detalhamento das informações referentes a um ou mais incidentes a serem notificados. A organização e a digitação do formulário serão feitas pelo gestor, e as informações referentes ao paciente e incidente serão realizadas pelo notificador. O formulário precisa ser preenchido completamente, o profissional pode notificar mais de um incidente e utilizar a caixa de texto para a descrição da ocorrência, que deve ser detalhada, clara e concisa. O instrumento é autoexplicativo, porém será disponibilizado um guia orientador para o preenchimento de dados do paciente e incidente no sistema interno on-line.

No Quadro 2, verificam-se os tipos de incidentes descritos no instrumento, complementado pelo campo "outros", no qual é possível incluir incidentes que não estão listados.

Quadro 2 - Notificações de incidentes mais frequentes Selecionar com 'X' um ou mais incidentes, como observado no Quadro 1.

\begin{tabular}{|l|l|}
\hline & Paciente não identificado ou falha na identificação \\
\hline & Falha na comunicação \\
\hline & Troca ou uso inadvertido de medicação \\
\hline & Troca de paciente ou sítio cirúrgico \\
\hline & Infecção relacionada ao cuidado \\
\hline & Úlcera por pressão \\
\hline & Queda ou risco de queda \\
\hline & Evento adverso \\
\hline & Incidente sem dano \\
\hline & Circunstâncias notificáveis \\
\hline & Quase falha \\
\hline & Outros \\
\hline
\end{tabular}

Fonte: Autoras.

Após o preenchimento das notificações evidenciadas no Quadro 2, é importante "sair" ou fechar o sistema para obter perfeita funcionalidade e segurança do instrumento. A última ação, "gravar", permite o envio imediato do formulário aos membros responsáveis designados a exercer funções no Núcleo de Segurança do Paciente. Ao final, deve-se aguardar a mensagem de confirmação aparecer para garantir que os dados foram gravados. 
Compõem as varáveis de interesse para notificação os itens a seguir:

1. Nome completo do paciente;

2. Número do prontuário;

3. Enfermaria e leito - utilizar barra entre as informações;

4. Data da internação;

5. Data da ocorrência;

6. Assinalar com "o" a faixa etária;

7. Assinalar com "o" para identificar o sexo;

8. Grade para marcar " $\mathrm{x}$ ";

9. Espaço para descrever o incidente;

10. Identificação do notificador;

11. Contato, e-mail e telefone;

12. Cargo ou função.

Após as análises e modelagens selecionadas para a formatação atual do formulário on-line, foi realizado um teste na instituição com o formulário apresentado na Figura 2.

Figura 2 - Formulário On-line.

\section{Notificação de Incidentes - FORMSUS}

DÚVIDAS E ORIENTAÇÕES PARA PREENCHIMENTO: RAMAIS XXXX- XXXX

*Preenchimento obrigatório

Atenção: Nos campos marcados com "visível ao público", não devem ser colocados dados de sua intimidade e privacidade.

1) Nome completo do paciente:*

2) No do Prontuário:*

3) Enfermaria - Leito:

(Ex.: $520-2)$

4) Data da Internação:

5) Data da Ocorrência:* 
6) Idade:*
0-18
19-35
36-59
$>59$
Não se aplica

\section{7) Sexo:*}
Masculino
C. Feminino
C. Não se aplica

8) Tabela:*

Marque com X

$\Gamma$ 01 - Paciente não identificado ou falha na identificação

ए 02 - Falha na comunicação

ए 03 - Troca ou uso inadvertido de medicação

ए 04 - Troca de paciente ou sítio cirúrgico

ए 05 - Infecção relacionada ao cuidado

ए 06 - Úlcera por pressão

$\Gamma$ 07 - Queda ou risco de queda

Г 08 - Atraso no diagnóstico e tratamento

Г 09 - Falha no uso de hemocomponentes

Г 10 - Falha na utilização de equipamentos/insumos

Г 11 - Tempo de internação prolongado sem justificativa

Г 12 - Desabastecimento, incêndio, pane elétrica, vazamento

Г13-Outros

9) Descrição completa do Incidente:*

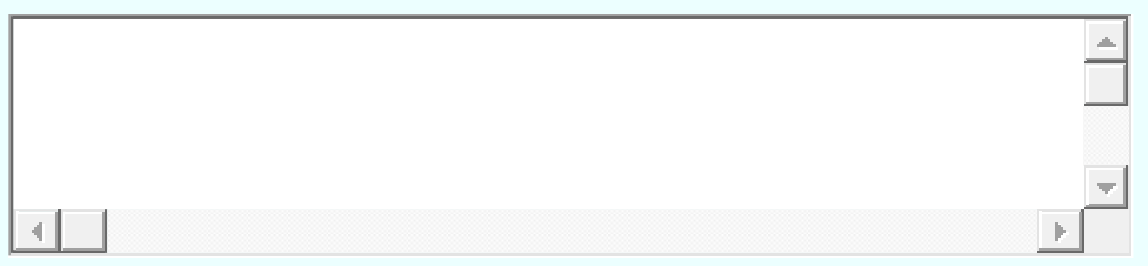


10) Notificador - Nome: (Opcional):

11) Contato (opcional): (e-mail/tel., etc.):

12) Cargo/Função (Obrigatório):*

\section{Gravar}

Fonte: Autoras.

Observa-se que o formulário apresentado na Figura 2 aprimora o processo de notificação de incidentes pela sua usabilidade. A estrutura do formulário oferece mais praticidade, tanto na inscrição dos eventos, quanto no acompanhamento da coleta de dados. Esse serviço on-line oferece uma visualização gráfica simples dos campos de preenchimento e um compartilhamento dos resultados de forma total ou restrita. A aplicação do formulário, conforme demonstrado, converge com as necessidades do mundo contemporâneo, pois adequa as necessidades do âmbito hospitalar ao contexto em que há a necessidade da viabilização e agilidade na troca de informações. A utilização do formulário precede definição sobre restrição de acesso: com acesso restrito a respondentes pré-cadastrados ou com acesso de login e senha, seguindo a prerrogativa da segurança e confidencialidade. Trata-se de uma ferramenta reativa para a gestão de risco, vigilância e monitoramento do que é notificado, que objetiva facilitar as notificações de incidentes no meio hospitalar e oferecer interação entre o profissional e o computador, de maneira que esse mecanismo possa ser um importante auxiliar no ambiente de trabalho.

\section{Considerações Finais}

Com a estruturação do sistema on-line interno no ambiente de trabalho, intenta-se contribuir para o enfrentamento às barreiras existentes para notificação, simplificando e padronizando os métodos, ampliando as possibilidades de adesão dos profissionais no processo de notificações de incidentes. O processo de desenvolvimento do instrumento demonstrou que este proporciona facilidade no acesso, agilidade e economia na realização e solução das demandas solicitadas. Desta forma, a instituição poderá, por meio de um sistema interno on-line para notificar incidentes ao Núcleo de Segurança do Paciente, identificar os incidentes, reduzir a ocorrência de erros e utilizar estratégias para melhorar a qualidade para um cuidado mais seguro.

\section{Referências}

Agência Nacional de Vigilância Sanitária. (2013). Resolução-RDC n. 36, de 25 de julho de 2013. Institui ações para a segurança do paciente em serviços e saúde e dá outras providências.

Banta, D., \& Almeida, R. T. (2009). The development of health technology assessment in Brazil. International journal of technology assessment in health care, 25(S1), 255-259.https://doi.org/10.1017/s0266462309090722

Departamento de Informática do SUS (DATASUS). (2021). FormSUS. http://datasus1.saude.gov.br/informacoes-de-saude/servicos2/formsus 
Hsia, T. L., Chiang, A. J., Wu, J. H., Teng, N. N., \& Rubin, A. D. (2019). What drives E-Health usage? Integrated institutional forces and top management perspectives. Computers in Human Behavior, 97, 260-270. https://doi.org/10.1016/j.chb.2019.01.010

Instituto Brasileiro de Geografia e Estatística. (2009). O Setor de Tecnologia da Informação e Comunicação no Brasil (Série estudos e pesquisas, informação econômica, n. 11).

Lima, H. F., Bezerra, C. E., \& Amaral, J. A. (2020). Organizando a pesquisa acadêmica: reflexões teórico-práticas sobre a metodologia dos trabalhos científicos. Research, Society and Development, 9(8), e227985166. http://dx.doi.org/10.33448/rsd-v9i8.5166

Meyer-Massetti, C., Cheng, C. M., Schwappach, D. L., Paulsen, L., Ide, B., Meier, C. R., \& Guglielmo, B. J. (2011). Systematic review of medication safety assessment methods. American Journal of Health-System Pharmacy, 68(3), 227-240. https://doi.org/10.2146/ajhp100019

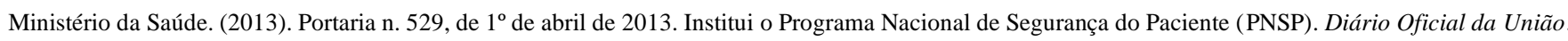

Ministério da Saúde. (2015a). FormSUS: Manual do Gestor. Ministério da Saúde. https://central3.to.gov.br/arquivo/257754/

Ministério da Saúde. (2015b). Relatório de Gestão do Exercício de 2014. http://portalarquivos.saude.gov.br/images/pdf/2015/maio/04/relatorio-gestao-sgepdatasus-2014.pdf

Ministério da Saúde. (2017). Estratégia e-Saúde para o Brasil. https://saudedigital.saude.gov.br/wp-content/uploads/2020/02/Estrategia-e-saude-para-oBrasil_CIT_20170604.pdf

Moraes, A., \& Mont'Alvão, C. (2003). Ergonomia: Conceitos e Aplicações, (3rd ed.), Luser.

Novoa, C. G., \& Valerio Netto, A. (2019). Fundamentos em gestão e informática em saúde. Escola Paulista de Medicina, Universidade Federal de São Paulo. p. 77. https://repositorio.unifesp.br/handle/11600/51788

Organização Mundial da Saúde; União Internacional das Telecomunicações. (2012). Pacote de Ferramentas da Estratégia Nacional de Saúde. https://saudedigital.saude.gov.br/wp-content/uploads/2020/02/Pacote-de-Ferramentas-da-Estrat\%C3\%A9gia-Nacional-de-e-Sa\%C3\%BAde.pdf.

Santa Rosa, J. G., \& de Moraes, A. (2008). Avaliação e projeto no design de interfaces. $2 \mathrm{AB}$.

Sousa, P., Lage, M. J., \& Rodrigues, V. (2019). Magnitude do problema e os factores contribuintes do erro e dos eventos adversos. Segurança do Paciente: conhecendo os riscos nas organizações de saúde. http://books.scielo.org/id/tzvzr/pdf/sousa-9788575416419-08.pdf

Wachter, R. M., \& Gupta, K. (2018). Understanding patient safety. McGraw-Hill Medical.

World Health Organization. (2009). Conceptual Framework for the International Classification for Patient Safety. https://www.who.int/patientsafety/taxonomy/icps_full_report.pdf 